01,07

\title{
Динамические смещения атомов и атермическое скольжение дислокаций в кристаллах
}

\author{
(C) Ю.A. $\mathrm{XoH}^{1}$, H. Zapolsky ${ }^{2}$ \\ ${ }^{1}$ Институт фризики прочности и материаловедения СО РАН, \\ Томск, Россия \\ ${ }^{2}$ University of Rouen Normandy, \\ Rouen, France \\ E-mail: khon@ispms.tsc.ru
}

Поступила в Редакцию 20 июня 2019 г.

В окончательной редакции 20 июня 2019 г.

Принята к публикации 27 ноября 2019 г.

Предложен механизм скольжения дислокации при низких температурах. В основе механизма лежит учет динамических смещений атомов - смещений вызванных неадиабатическими переходами атомов в кристалле с дислокацией под действием внешней силы. Динамические смещения инициируют неустойчивость прямой дислокации относительно малоамплитудных смещений при колебаниях атомов. Развитие неустойчивости приводит к образованию двойного кинка и смещению дислокации на одно межатомное расстояние.

Ключевые слова: дислокация, динамические смещения, динамическая неустойчивость, атермическое скольжение дислокации.

DOI: 10.21883/FTT.2020.04.49112.533

\section{1. Введение}

Согласно классическим представлениям дислокационная пластичность кристаллов определяется механизмом термически активированного движения дислокации в потенциальном рельефе Пайерлса, состоящем из долин и потенциальных барьеров [1]. Высота потенциального барьера определяет энергию активации и напряжение Пайерлса $\tau_{P}$. Движение дислокации в рельефе Пайерлса происходит путем образования двойного кинка и его распространения вдоль линии дислокации. При напряжении $\tau_{P}$ дислокация с двойным кинком находится на вершине потенциального барьера. Напряжение Пайерлса может быть вычислено в хорошем согласии с экспериментальными данными [2]. Что касается механизма образования двойного кинка, то здесь ситуация более сложная. Существует устойчивое мнение, что образование двойного кинка определяется термически активированными смещениями атомов. Этот механизм подтверждается расчетами методом молекулярной динамики [3,4], но только при температуре, превышающей несколько десятков Кельвин. При температуре порядка нескольких Кельвин из-за малой амплитуды термических флуктуаций время ожидания крупной флуктуации становится неприемлемо большим. В то же самое время движение дислокаций наблюдается экспериментально и при низких температуpax, в частности, при температуре жидкого гелия [5-8]. При этом скорости деформации образца и величины деформирующих напряжений остаются примерно теми же, что и при повышенных температурах. Для объяснения атермического скольжения дислокации предложен механизм, определяемый туннелированием атомов через потенциальный барьер [9]. Но вероятность проявления этого механизма для атомов, содержащих в ядрах десятки и сотни нуклонов, чрезвычайно мала. Таким образом, должны существовать другие механизмы атермического скольжения дислокации. Один из них рассматривается ниже.

\section{2. Модель атермического скольжения дислокации}

Кристалл, в котором под действием внешней силы движется дислокация, представляет открытую нестационарную систему ядер и электронов. Динамика ядер в таких системах является неадиабатической [10]. Дело в том, что потенциальная энергия системы ядер и электронов состоит из множества пересекающихся листов. Каждый лист соответствует определенному квантовому состоянию кристалла (распределению ядер и электронов). Эти состояния могут быть разделены потенциальным барьером. Если не рассматривать туннелирование через потенциальный барьер, то известны два механизма перехода из одного состояния в другое. В первом механизме потенциальный барьер преодолевается за счет термически активированных смещений атомов. Наличие потенциального барьера приводит к необратимому смещению дислокации. Второй механизм определяется безбарьерными неадиабатическими переходами атомов между различными листами гиперповерхности потенциальной энергии (проявление эффекта Ландау-Зинера $[11,12])$. Листы различаются распределением электронной плотности и, как следствие, параметрами межатомного взаимодействия. 
На атомном уровне неадиабатические переходы проявляются в возбуждении кластеров с топологическим ближним порядком в распределении атомов, нехарактерным для изолированного кристалла. Такой тип ближнего порядка был назван динамическим ближним порядком [13]. Изменение динамического ближнего порядка сопровождается смещениями атомов, отличающимися от термически активированных смещений. Их для определенности будем также называть динамическими. Естественно, что возбуждаются такие динамические смещения, которые приводят к меньшему значению потенциальной энергии системы при той же самой величине деформации кристалла. В результате дислокация испытывает дополнительные неупругие обратимые смещения. Следует заметить, что область неупругой обратимой деформации всегда присутствует на кривых „напряжение-деформация“ кристалла и отделяет область упругой деформации от необратимой деформации. Это означает, что по мере увеличения нагрузки упруго деформированный кристалл вначале становится неустойчивым относительно динамических, и лишь затем относительно термически активированных смещений атомов.

Характерные размеры дислокации (сотни-тысячи атомов) и характерные времена деформации кристалла (доли секунды-минуты) делают решение задачи о зарождении двойного кинка методом неадиабатической молекулярной динамики практически невыполнимой. В этой ситуации можно воспользоваться подходами, развитыми в теории нелинейных систем [14,15], основанными на рассмотрении вблизи порога устойчивости решений линеаризованных уравнений движения медленно меняющихся в пространстве и времени амплитуд неустойчивых мод. В таком континуальном приближении дислокация рассматривается как тонкая нить, форма и размеры которой меняются вследствие термически активированных и динамических смещений атомов.

Пусть в кристалле без нагрузки (в исходном состоянии) прямая нерасщепленная дислокация расположена на дне долины Пайерлса с координатой $y=0$ в плоскости скольжения $x y$ декартовой системы координат. Линия дислокации направлена вдоль оси $x$. Потенциальная энергия кристалла в этом состоянии имеет локальный минимум. Потенциальная энергия кристалла с дислокацией в соседней долине Пайерлса (в точке $y=b$ ) также имеет локальный минимум. Эти два состояния разделены потенциальным барьером, высота которого равна $U_{P}$. Под действием приложенного напряжения $\tau=\dot{\tau} t$ (здесь $\dot{\tau}-$ скорость изменения напряжения, $t-$ время) кристалл деформируется, атомы смещаются из положения равновесия. Начальное состояние становится метастабильным, конечное - стабильным.

Ниже порога устойчивости $\tau_{d}$ дислокация деформируется упруго, величина смещений равна $y_{e l}(x, t)$. Вблизи порога устойчивости на фоне упругих смещений атомов возбуждаются динамические смещения атомов, приводящие к неупругому обратимому смещению дислокации на величину $b q(x, t)$, где $q-$ безразмерная амплитуда динамических смещений. Величина $q$ прямо пропорциональна объемной доле кластеров динамического ближнего порядка и меняется в интервале $0 \leq q \leq 1$. На пороге устойчивости $q=0$. В дальнейшем для краткости амплитуду $q$ будем называть динамической. При дальнейшем увеличении напряжения на фоне упругих и динамических смещений атомов возбуждаются термически активированные смещения $b \eta(x, t)$ с безразмерной амплитудой $0 \leq \eta \leq 1$. При смещении дислокации на одно межатомное расстояние $\eta$ принимает значение, равное единице.

Уравнение эволюции для амплитуды $\rho(\rho=q, \eta)$ представляет уравнение Ландау-Халатникова

$$
\frac{\partial \rho}{\partial t}=-\Gamma_{\rho} \frac{\delta F_{\rho}}{\delta \rho}
$$

где $\Gamma_{\rho}-$ кинетический коэффициент, $F_{\rho}=\int f_{\rho}(q, \eta, \tau) d V$ функционал, имеющий смысл свободной энергии с плотностью $f_{\rho}$, интегрирование ведется по объему системы $V$. Процессы возбуждения динамических и термически активированных смещений разнесены по времени. Это означает, что характерное время возбуждения динамических смещений $t_{q}$ намного меньше характерного время возбуждения термически активированных смещений $t_{\eta}$. При этом $t_{q}$ от температуры не зависит. В области неупругой обратимой деформации плотность свободной энергии $f_{q}$ представим в виде

$$
f_{q}(q, \eta, \tau)=f_{e l}(\tau)+\varphi_{q}(\eta, q, \tau) .
$$

Здесь $f_{e l}-$ плотность упругой энергии при $\tau<\tau_{d}$, $\varphi_{q}$ - вклад, определяемый динамическими смещениями при $\tau>\tau_{d}$. Используя разложение Гинзбурга-Ландау, представим функцию $\varphi_{q}$ в виде

$$
\begin{aligned}
\varphi_{q}(q, \eta, \tau)= & -\frac{A_{q}(\eta, \tau) q^{2}}{2}+\frac{C_{q}(\eta, \tau) q^{4}}{4} \\
& +G_{q}(\eta, \tau)(\nabla q)^{2} / 2,
\end{aligned}
$$

где $A_{q}>0, C_{q}>0, G_{q}>0-$ коэффициенты разложения. Знак „-“ перед $A_{q}$ означает, что динамические смещения атомов понижают энергию системы. Квантовое происхождение динамических смещений предполагает отсутствие потенциального барьера (кубических членов в разложении (2)). С учетом (2) уравнение эволюции для $q$ принимает вид

$$
t_{q} \frac{\partial q}{\partial t}=\alpha(\eta, \tau) q-q^{3}+l_{q}^{2} \Delta q
$$

Здесь параметры $t_{q}=\Gamma_{q} C_{q}, l_{q}=(G / C)^{1 / 2}$ имеют смысл характерного времени и характерной длины изменения амплитуды $q$ соответственно. Коэффициент $\alpha(\eta, \tau)=A_{q}(\eta, \tau) / C_{q}(\eta, \tau)$. В линейном приближении

$$
\alpha(\eta, \tau)=\alpha(\tau)-c \eta
$$

где $\alpha \geq 0$ и растет с увеличением $\tau$. Положительный знак коэффициента связи $c$ означает, что некогерентные 
термические флуктуации атомов уменьшают вклад динамических смещений в процессы релаксации. В равновесном состоянии уравнение (3) должно иметь единственное решение $q=0$. Это имеет место при $c \geq \alpha$. Учитывая квантовое происхождение динамических смещений, все параметры в (3), (4) от температуры явно не зависят.

Равновесное значение амплитуды $\eta$ определяется из условия минимума $f_{\eta}$ при заданных значениях $\tau$ и $q$. Эта функция должна иметь минимумы в точках $\eta=0$, 1 и максимум в точке $0 \leq \eta_{1} \leq 1$. Тогда разложение Гинзбурга-Ландау функции $f_{\eta}$ по $\eta$ и градиенту $\eta$ принимает вид

$$
\begin{aligned}
f_{\eta}(\eta, q, \tau)= & A_{\eta}\left\{\left[\frac{\eta_{1} \eta^{2}}{2}-\frac{\left(1+\eta_{1}\right) \eta^{3}}{3}+\frac{\eta^{4}}{4}\right]\right. \\
& \left.+\left(\frac{G_{\eta}}{2 A_{\eta}}\right)(\nabla \eta)^{2}\right\}+f_{\eta}(\eta, q, \tau) .
\end{aligned}
$$

Коэффициенты разложения $A_{\eta}>0, G_{\eta}>0$ считаются постоянными, $\eta_{1}=\eta_{1}(q, \tau)$. С учетом (5) кинетическое уравнение для $\eta$ принимает вид

$$
t_{\eta} \frac{\partial \eta}{\partial t}=-\eta_{1} \eta+\left(1+\eta_{1}\right) \eta^{2}-\eta^{3}+l_{\eta}^{2} \Delta \eta
$$

Здесь $t_{\eta}=\Gamma_{\eta} A_{\eta}, \quad l_{\eta}=\left(G_{\eta} / A_{\eta}\right)^{1 / 2}$ имеют смысл характерного времени и длины изменения амплитуды $\eta$ соответственно. В теории термически активированных процессов принимается, что

$$
t_{\eta}=t_{\eta 0} \exp \left(\frac{U_{P}-W_{\tau}}{k_{\mathrm{B}} T}\right),
$$

где $k_{\mathrm{B}}-$ постоянная Больцмана, $W-$ активационный объем, $t_{\eta 0}$ - величина, обратно пропорциональная частоте колебаний. Высота потенциального барьера, разделяющего два равновесных состояния прямой дислокации, определяется значением функции $f_{\eta}\left(\eta_{1}\right)$. При $\eta_{1}=0 f_{\eta}(0)=0$. В линейном приближении

$$
\eta_{1}(q, \tau)=\eta_{10}-d q
$$

Здесь $\eta_{10}=\eta_{10}(\tau)$ значение $\eta_{1}$ при $q=0$. Коэффициент связи $d>0$. Знак „-““ означает, что проявляются только те динамические смещения, которые понижают плотность свободной энергии.

\section{3. Зарождение двойного кинка и атермическое смещение дислокации}

Система связанных уравнений (3), (4), (6)-(8) описывает кинетику зарождения двойного кинка, его развитие и смещение дислокации на одно межатомное расстояние при различных температурах $T$ и величины приложенного напряжения. Удобно рассмотреть вначале стационарные однородные решения уравнений (3), (4),
(6)-(8) соответствующие прямой дислокации. В области $0 \leq \eta \leq 1,0 \leq q<1$ имеются четыре однородных решения $(\eta, q)$ : 1. $(0,0) ; 2 .(1,0) ; 3 .\left(0, q_{s}\right) ; 4 .(\alpha / \beta, 0)$. Здесь

$$
q_{s}=\sqrt{\alpha}
$$

Четвертое решение особого интереса не представляет. Стандартный анализ устойчивости однородных решений относительно малых однородных и неоднородных возмущений $\delta \eta, \delta q \sim \exp (\gamma t+k x)(k-$ волновое число) показывает следующее. Решение $(0,0)$ неустойчиво относительно малых возмущений $\delta q(\operatorname{Re} \gamma>0)$. Решение $(1,0)$ устойчиво относительно малых однородных и неоднородных возмущений $(\operatorname{Re} \gamma<0)$. Решение $\left(0, q_{s}\right)$ при

$$
d \sqrt{\alpha}>\eta_{10}
$$

неустойчиво относительно малых возмущений, а при

$$
d \sqrt{\alpha}<\eta_{10}
$$

устойчиво относительно малых однородных и неоднородных возмущений, но неустойчиво относительно возмущений с амплитудой

$$
\delta \eta>\delta \eta_{c}=\eta_{10}-d \sqrt{\alpha} .
$$

Причина неустойчивости решения $\left(0, q_{s}\right)$ следует из того, что коэффициент перед $\eta$ в правой части (6) при выполнении условия (10) становится положительным, и сколь угодно малое возмущение - нарастает. Неоднородные решения уравнений (3), (4), (6)-(8) находились численными методами. Метод решения, использованные начальные, граничные условия и начальные возмущения приведены в Приложении.

Пусть при низких температурах $0<\eta_{10}<1 / 2$. Дислокация расположена в исходной долине Пайерлса. Соответствующее этому положению решение $\eta=0, q=0$. Отрицательный и положительный знаки первых слагаемых в правых частях (3), (6) соответственно приводят к тому, что малые возмущения $\delta_{\eta}$ затухают, а $\delta q-$ нарастают до стационарного значения $q_{s}=\sqrt{\alpha}$. На рис. 1 приведены пространственные зависимости динамической амплитуды в различные моменты времени для случая, когда условие (10) не выполняется. Видно образование двойного перегиба, и его расширение в виде двух волн переключения $q=q\left(x \mp v_{q} t\right)$ со скоростью $v_{q} \approx l_{q} / t_{q}$. Увеличение размера области стационарных значений $q$ приводит к неупругой деформации дислокации. При увеличении $\tau$ до значений $\tau_{P}$, соответствующих выполнению условия (10), первое слагаемое в правой части (6) становится положительным. Решение $\left(0, q_{s}\right)$ становится неустойчивым относительно флуктуаций $\delta_{\eta}$ со сколь угодно малой амплитудой. Это могут быть и нулевые колебания. Образуется двойной кинк (рис. 2). Его расширение происходит в виде двух волн переключения $\eta=\eta\left(x \mp v_{\eta} t\right)$, распространяющихся в противоположные стороны. При $\eta \rightarrow 1 q \rightarrow 0$. Однородное решение $(1,0)$ устойчиво, дислокация оказывается в соседней 
долине. На рис. 3 приведены пространственные распределения переменных $\eta, q$ в разные моменты времени для случая, когда концы дислокации закреплены. Видно, что, как и на рис. 2, образуется двойной кинк, и часть дислокации смещается в соседнюю долину Пайерлса.

Если следить за динамикой движения дислокации in situ, то картина движения дислокации будет выглядеть следующим образом. При $\tau<\tau_{d}$ петля дислокации изгибается упруго. При $\tau>\tau_{d}$ дислокация испытывает дополнительные неупругие смещения в направлении действия приложенной силы. Чем больше напряжение, тем больше величина неупругой деформации дислокации. При увеличении длины дислокации примерно

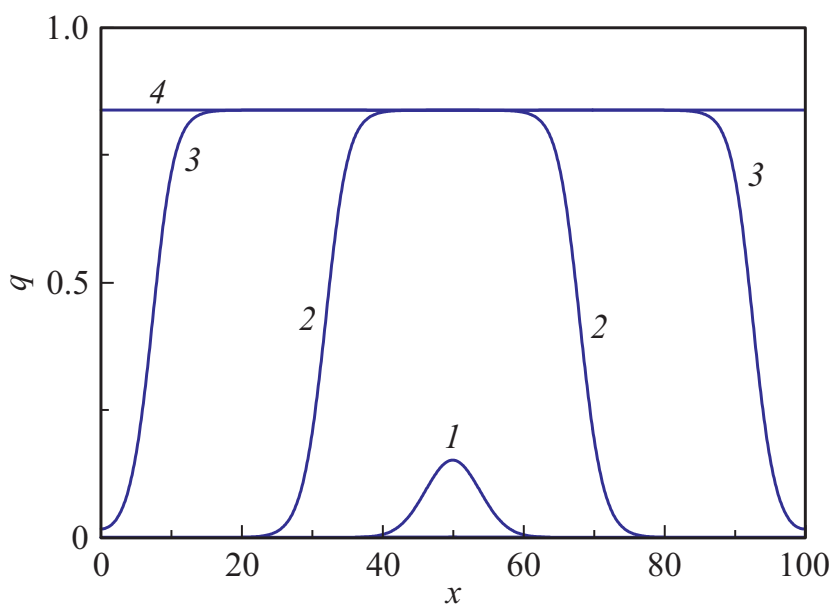

Рис. 1. Пространственное распределение переменной $q$ в моменты времени $t=5,20,35,55$ (кривые 1,2, 3, 4 соответственно) при граничных условиях (А2), (А3), начальных условиях (А5), начальном возмущении (А8), (А10). Параметры системы уравнений (3), (4), (6)-(8) указаны в (A11).

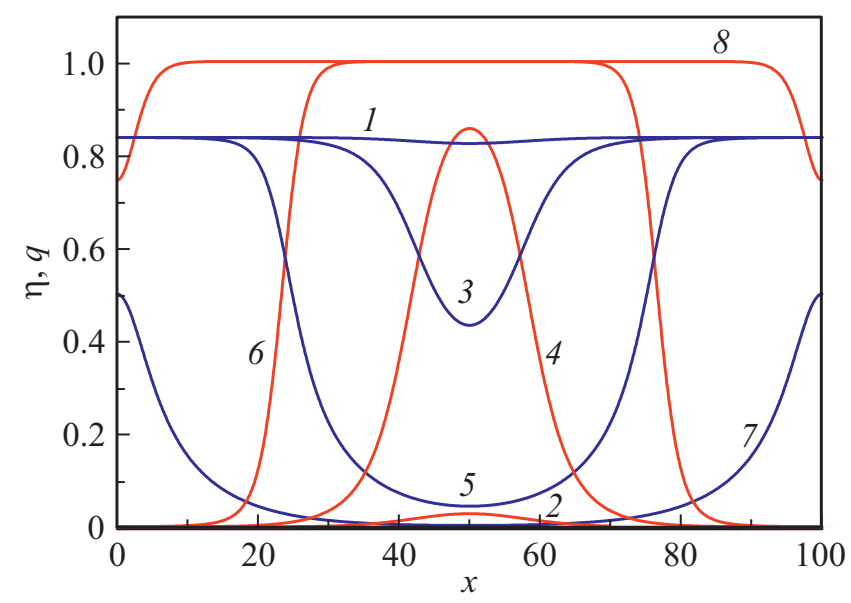

Рис. 2. Пространственное распределение переменных $q$ (кривые $1,3,5,7)$ и $\eta$ (кривые 2, 4, 6, 8) в моменты времени $t=30$ (кривые 1,2), 60 (кривые 3,4), 80 (кривые 5,6), 110 (кривые 7,8) при граничных условиях (А2), (А3), начальных условиях (А6), начальном возмущении для $\eta$ (A7), (A12), начальном возмущении для $q$ (А9). Параметры системы уравнений (3), (4), (6)-(8) указаны в (А13).

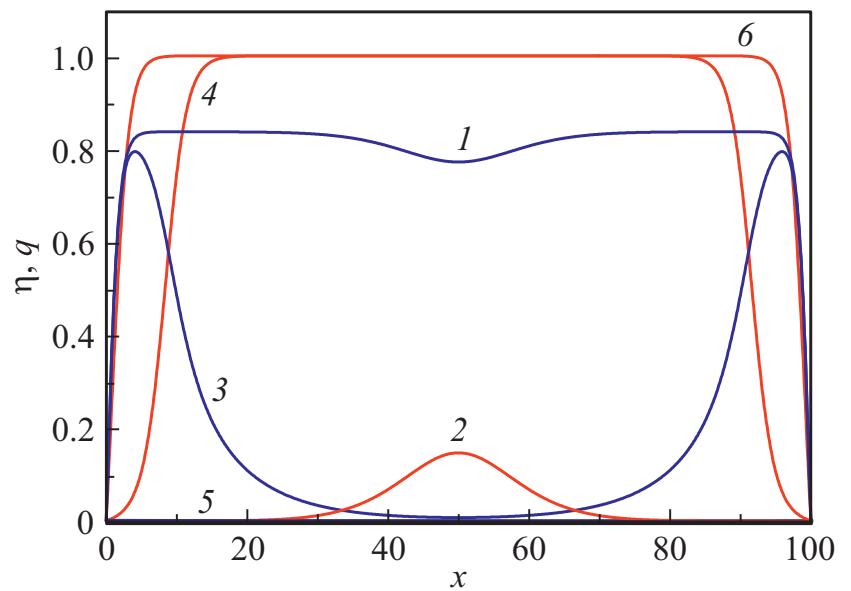

Рис. 3. Пространственное распределение переменных $q$ (кривые $1,3,5)$ и $\eta$ (кривые 2,4,6) в моменты времени $t=50$ (кривые 1,2), 100 (кривые 3,4), 110 (кривые 5, 6) при граничных условиях (А2), (А4), начальных условиях (А6), начальном возмущении для $\eta(\mathrm{A} 7),(\mathrm{A} 12)$, начальном возмущении для $q$ (А9). Параметры системы уравнений (3), (4), (6)-(8) указаны в (А13).

на два межатомных расстояния образуется состояние, неустойчивое относительно флуктуаций при колебаниях атомов с малой амплитудой. Развитие неустойчивости приводит к зарождению и движению двойного кинка. Внешне картина будет выглядеть так, как будто крупная флуктуация возникла за счет коллективного смещения атомов при нулевых колебаниях атомов. Но без учета динамических смещений атомов для смещения дислокации требуются более высокие напряжения по сравнению с напряжением Пайерлса.

\section{4. Заключение}

В основе решения задачи об атермическом скольжении дислокации лежит рассмотрение кристалла с дислокацией при деформации как открытой нестационарной системы. В таких системах помимо смещений, определяемых величиной внешней силы и термическими флуктуациями при колебаниях атомов, могут возбуждаться динамические смещения. Эти смещения обусловлены изменением топологического ближнего порядка при неадиабатических переходах атомов между различными квантовыми состояниями кристалла. Возбуждение динамических смещений не зависит от температуры и проявляется в неупругом обратимом удлинении дислокации. Возрастание величины неупругой обратимой деформации вызывает неустойчивость системы относительно флуктуаций с малой амплитудой при колебаниях атомов. Развитие неустойчивости сопровождается образованием двойного кинка, его движением вдоль дислокации, и ее смещением на межатомное расстояние. 


\section{Благодарности}

Авторы благодарят Л.Б. Зуева, П.П. Каминского, Е.Е. Слядникова за интерес к работе и полезные замечания.

\section{Финансирование работы}

Работа выполнена в рамках Программы фундаментальных научных исследований государственных академий наук на 2013-2020 годы, направление III.23.

\section{Конфликт интересов}

Авторы заявляют, что у них нет конфликта интересов.

\section{Приложение}

Численное решение уравнений (3), (4), (6)-(8) проводилось по неявной схеме. Вводились безразмерные переменные

$$
x=x / l_{\eta}, \quad t=t / t_{\eta}, \quad l=l_{q} / l_{\eta}, \quad \theta=t_{q} / t_{\eta}
$$

Граничные условия:

$$
\begin{gathered}
0 \leq x \leq X, \quad X=100 . \\
\left.\frac{d \eta}{d x}\right|_{x=0}=\left.\frac{d \eta}{d x}\right|_{x=X}=0,\left.\quad \frac{d q}{d x}\right|_{x=0}=\left.\frac{d q}{d x}\right|_{x=X}=0, \\
q(x=0, t)=q(x=X, t)=0 \\
\eta(x=0, t)=\eta(x=X, t)=0 .
\end{gathered}
$$

Начальные условия:

$$
\begin{aligned}
& \text { 1. } \eta(x, t=0)=0, \quad q(x, t=0)=0 ; \\
& \text { 2. } \eta(x, t=0)=0, \quad q(x, t=0)=q_{s} .
\end{aligned}
$$

Локализованные возмущения задавались в виде

$$
\begin{aligned}
& \Delta \eta=\Delta \eta_{0} \exp \left[-\sigma_{\eta}\left(x-x_{0}\right)^{2}\right], \\
& \Delta q=\Delta q_{0} \exp \left[-\sigma_{q}\left(x-x_{0}\right)^{2}\right],
\end{aligned}
$$

где $\Delta \eta_{0}, \Delta q_{0}$ - амплитуды, $x_{0}$ - начальное положение, $\sigma_{\eta}, \sigma_{q}$ - дисперсии возмущений переменных $\eta, q$ соответственно.

Стохастическое возмущение $\Delta q$ в каждом узле расчетной сетки принимало случайное значение в интервале

$$
\begin{gathered}
0 \leq \Delta q(x) \leq 0.001 . \\
\Delta q_{0}=0.01, \quad \sigma_{q}=5, \quad x_{0}=50 . \\
\eta_{1}=0.2, \quad d=0.3, \quad \alpha=0.3, \quad c=0.8 . \\
\Delta \eta_{0}=0.01, \quad \sigma_{\eta}=15, \quad x_{0}=50 . \\
\eta_{1}=0.2, \quad d=0.3, \quad \alpha=0.7, \quad c=0.8 .
\end{gathered}
$$

\section{Список литературы}

[1] R.E. Peierls. Proc. Phys. Soc. Lond. 52, 34 (1940).

[2] L. Proville, D. Rodney, M.-C. Marinica. Nature Mater. 11, 845 (2012).

[3] S. Narayanan, D.L. McDowell, T. Zhu. J. Mech. Phys. Solids 65, 54 (2014).

[4] C. Domain, G. Monnet. PRL 95, 215506 (2005).

[5] T.H. Blewitt, J.R. Redman, T.A. Sherill, R.R. Coltman. Phys. Rev. 98, 1555 (1955).

[6] E.T. Wessel. Trans. ASM. 49, 149 (1957).

[7] Z.S. Basinski, A. Sleeswyk. Acta Met. 5, 3, 176 (1957).

[8] P. Haasen. Phil. Mag. 3, 384 (1958).

[9] B.V. Petukhov, V.L. Pokrovskii. Sov. Phys. JETP. 36, 2, 336 (1973).

[10] R. Kapral. J. Phys.: Condens. Matter. 27, 073201 (2015).

[11] L. Landau. Phys. Z. Sow. 2, 46 (1932).

[12] C. Zener. Proc. R. Soc. A 137, 696 (1932).

[13] V.E. Egorushkin, N.V. Mel'nikova. J. Exp. Theor. Phys. 76, 103 (1993).

[14] M.C. Cross, P.C. Hohenberg. Rev. Mod. Phys. 65, 854 (1993).

[15] I.S. Aranson. Rev. Mod. Phys. 74, 99 (2002).

Редактор Т.Н. Василевская 